\title{
Implementing near-peer learning: a resuscitation skills development module for medical students in middle income countries
}

Kaushila Thilakasiri ${ }^{1}$,Praveen Weeratunga ${ }^{2}$, Prasanna Nadarajah ${ }^{1}$, N.A. Sandaruwan Kanchana ${ }^{1}$, Chathurani Sigera ${ }^{3}$

1Post Graduate Institute of Medicine University of Colombo, 2 Faculty of Medicine, University of Colombo, 3 Institute of Health Policy Sri Lanka

\section{Purpose of the Study}

Most medical curricula in low and middle income countries still utilize traditional, didactic, lecturer led approaches to resuscitation training. Utilization and research on near-peer learning (NPL) in lower-middle income countries (LMICs) is limited. This study evaluates the effectiveness of a skills training course for final year medical students, developed and delivered by postgraduate trainees in emergency medicine (Registrars).

\section{Material and Method}

Twelve registrars, all whom were instructors or candidate instructors of ERC ALS course participated as instructors voluntarily to maintain $1: 5$ instructor to participant ratio on each day. A convenient sample of 105 (37 female, 68 males) final year medical students were selected. Pre- reading material which was based on ERC guideline was provided 1 week prior. A pretest of $12 \mathrm{MCQ}$ questions were given just prior to beginning the course. A student group comprising of 14 members were divided in to three smaller groups to participate in 30 minute skills stations on ABCDE assessment, BLS and Safe defibrillation, Airway management in cardiac arrest. ALS algorithm was demonstrated by the resource person to entire 14 member group.

\section{Results}

Pre and post test marks show a statistically significant improvement in knowledge; $77.7 \%$ to $88.83 \%$ ( $p<0.01$ ). Level of confidence in performing skills improved from 3 or below to 4 or above out of 5 in $64.76 \%$. Majority of participants rated the course as excellent or very good $(92 \%), 100 \%$ would recommend the course to peers, $50.47 \%$ strongly agreed that the course content was well planned and organized, learning environment was non threatening for $91.42 \%$. Instructors were rated as available and helpful by $98.09 \%$.Istructors stimulated student interest according to $89.5 \%$ and $88.57 \%$ reported that time was effectively utilized. Opportunity to practice a skill on a manikin was considered the most useful aspect of the course.

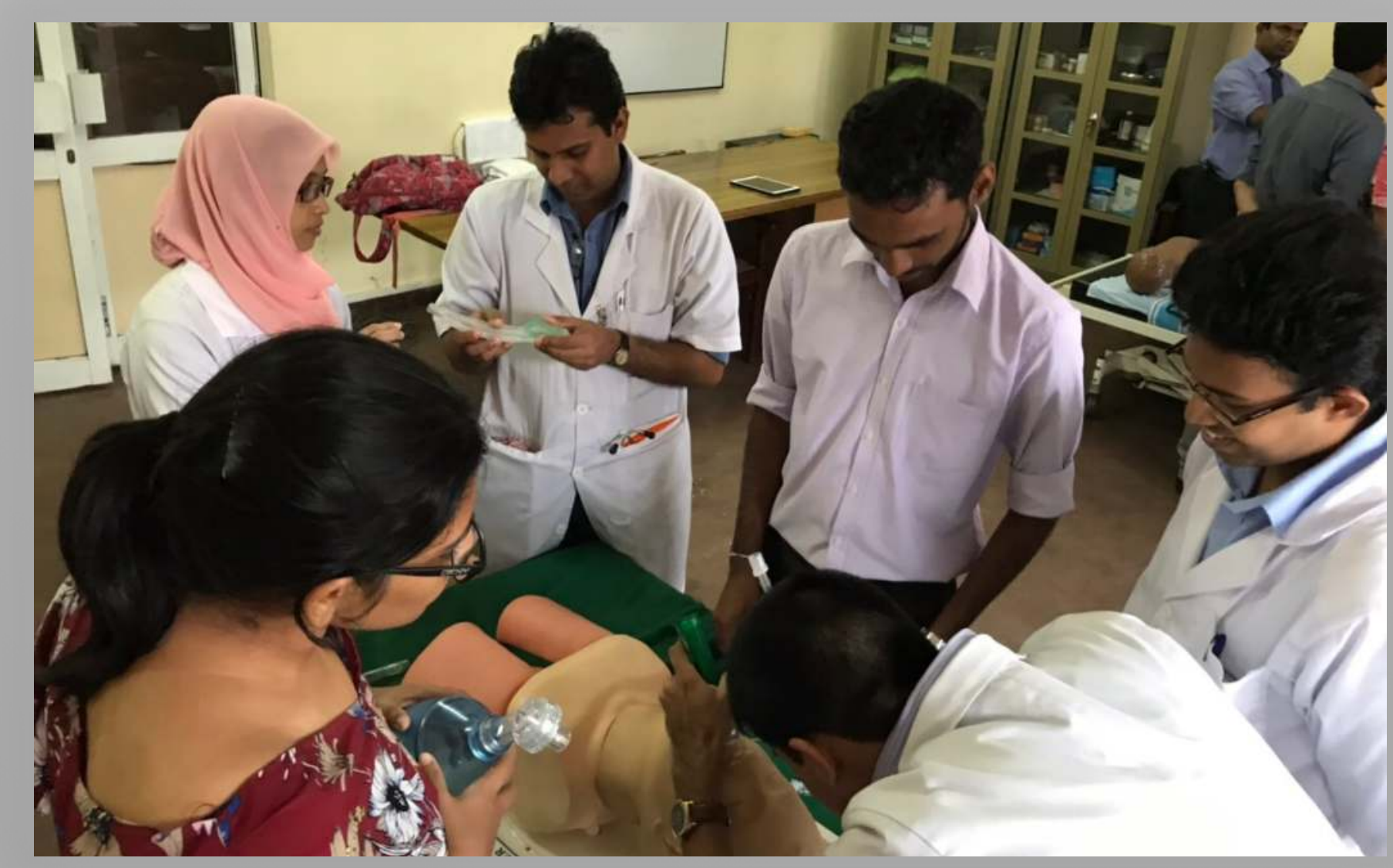

\section{Conclusion}

"Post graduate trainees in Sri Lanka have successfully developed and

implemented a cost effective, resuscitation knowledge and skills development course for medical students, creating a positive and safe learning environment."

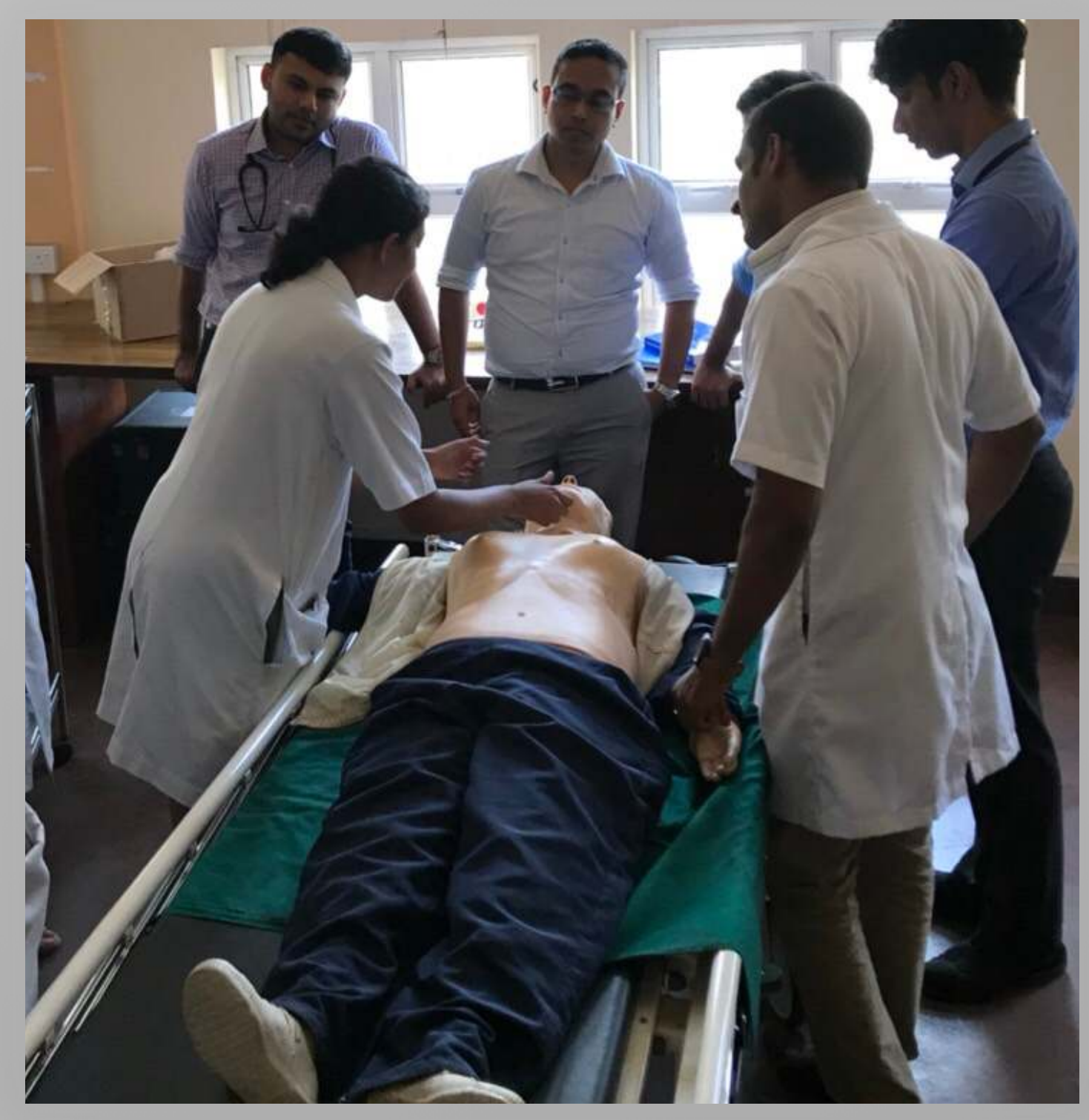

\section{Acknowledgement}

College of Anaesthesiologists \& Intensivists of Sri Lanka - Working Committee on Resuscitation lead by Dr. Mithrajee Premarathne and Dr.Nilmini Wijesuriya , University of Colombo Faculty of Medicine, Department of Clinical Medicine and skills lab, Marion De Almeida Santos at the Queen Elizabeth Hospital Birmingham, All the Emergency Medicine registrars who volunteered to teach skills; Achala, Kaminda, Isuru, Hasitha, Lanka, Nadini, Sabeer, Shiromi, Surangika, Thusitha, Varuna, Thushini, Kushan, Yasith 